\title{
Charmless three-body $B$ meson decays at $\mathrm{LHCb}$
}

\author{
Daniel O’Hanlon*广 \\ INFN Bologna \\ E-mail: dohanlondern.ch
}

\begin{abstract}
Multi-body $b$-hadron decays to charmless final states, are a particularly interesting place to search for violation of $C P$ symmetry, due to the difference in weak and strong phases in competing decay amplitudes to many charmless final states. Furthermore, the weak phase accessible in certain loop-domiated $B_{(s)}^{0} \rightarrow K_{\mathrm{S}}^{0} h^{+} h^{-}$decays is expected, in the Standard Model, to be similar to that of tree-dominated $b \rightarrow c \bar{c} s$ decays. Here measurements of the branching fractions of the $B_{(s)}^{0} \rightarrow$ $K_{\mathrm{S}}^{0} h^{+} h^{-}$decays are presented, which are the most precise measurements of these quantities todate. Furthermore, an amplitude analysis of the $B^{0} \rightarrow K_{\mathrm{S}}^{0} \pi^{+} \pi^{-}$decay is also presented, where $C P$ violation is observed in the $\bar{B}^{0} \rightarrow K^{*}(892)^{-} \pi^{+}$decay with a significance of approximately $6 \sigma$.
\end{abstract}

The 39th International Conference on High Energy Physics (ICHEP2018)

4-11 July, 2018

Seoul, Korea

* Speaker.

${ }^{\dagger}$ On behalf of the LHCb collaboration. 


\section{Introduction}

Decays of $b$-hadrons to charmless final states are a particularly interesting area to search for violation of charge-parity $(C P)$ symmetry in decay, where a difference in weak and strong phases between two competing amplitudes are required to generate observable $C P$ asymmetry. In charmless decays, the interfering tree and penguin amplitudes often carry different Cabibbo-KobayashiMaskawa (CKM) weak phases, and furthermore, multi-body decays give access to additional strong phases that vary as a function of the phase-space location that are often associated with the resonant structure. The neutral $B_{(s)}^{0} \rightarrow K_{\mathrm{S}}^{0} h^{+} h^{-}$decays ${ }^{1}$ are also of particular interest as the mixing-induced asymmetries in these loop-domiated decays are predicted in the Standard Model to be approximately equal to those governed by tree-level $b \rightarrow c \bar{c} s$ transitions. For certain intermediate decays, such as $B^{0} \rightarrow \phi K_{\mathrm{S}}^{0}$ and $B^{0} \rightarrow \rho^{0} K_{\mathrm{S}}^{0}$, it also predicted that the SM weak phase is similar to that obtained in $b \rightarrow c \bar{c} s$ decays, a difference that could be enhanced with the presence of new heavy particles participating in the loop-dominated charmless decays.

The LHCb detector [1] is a forward spectrometer, optimised for the study of $b$ - and $c$-hadron decays, and fully instrumented in the $2<\eta<5$ pseudorapidity region. Efficient discrimination of charged hadrons with momenta in the range $1-100 \mathrm{GeV}$ is achieved with two ring-imaging Cherenkov detectors. Decays of particles with a significant flight distance, such as $K_{\mathrm{S}}^{0}$ mesons, are reconstructed either with 'downstream' tracks, that are formed only of hits in tracking stations downstream of the vertex detector that surrounds the the interaction point, or with 'long' tracks, that are constructed using hits in all tracking stations.

\section{Measurement of the branching fractions of $B_{(s)}^{0} \rightarrow K_{\mathrm{S}}^{0} h^{+} h^{-}$decays}

The branching fractions of the $B_{(s)}^{0} \rightarrow K_{\mathrm{S}}^{0} h^{+} h^{-}$decays are measured using $3 \mathrm{fb}^{-1}$ of Run 1 LHCb data [2], including a search for the previously unobserved $B_{s}^{0} \rightarrow K_{\mathrm{S}}^{0} K^{+} K^{-}$decay, relative to the $B^{0} \rightarrow K_{\mathrm{S}}^{0} \pi^{+} \pi^{-}$decay. A multivariate classifier is used to suppress combinatorial background to the signal channels, the requirements of which are optimised separately for the favoured and suppressed modes to increase sensitivity. The combinatorial background that remains after this procedure, along with other sources of background, such as that from four-body decays and $\pi \leftrightarrow K$ mis-identification, are modelled along with the $B^{0}$ and $B_{s}^{0}$ signal shapes for each final state in a simultaneous maximum likelihood fit to extract the signal yields. The results of this can be seen for the $K_{\mathrm{S}}^{0} \pi^{+} \pi^{-}$final state in Figure 1, using the optimisation criteria for the favoured decay modes.

Efficiencies are calculated primarily using simulation, with data-driven corrections for the particle identification hardware trigger efficiencies, as a function of the two-dimensional Dalitz plot using the sPlot procedure [3]. Using the world-average value of the $B^{0} \rightarrow K_{s}^{0} \pi^{+} \pi^{-}$decay branching fraction [4], the absolute branching fractions are found to be

$$
\begin{aligned}
\mathscr{B}\left(B^{0} \rightarrow \bar{K}^{0} K^{ \pm} \pi^{\mp}\right) & =(6.1 \pm 0.5 \pm 0.7 \pm 0.3) \times 10^{-6}, \\
\mathscr{B}\left(B^{0} \rightarrow K^{0} K^{+} K^{-}\right) & =(27.2 \pm 0.9 \pm 1.6 \pm 1.1) \times 10^{-6}, \\
\mathscr{B}\left(B_{s}^{0} \rightarrow K^{0} \pi^{+} \pi^{-}\right) & =(9.5 \pm 1.3 \pm 1.5 \pm 0.4) \times 10^{-6},
\end{aligned}
$$

\footnotetext{
${ }^{1}$ Charged-conjugate decays are implied throughout.
} 

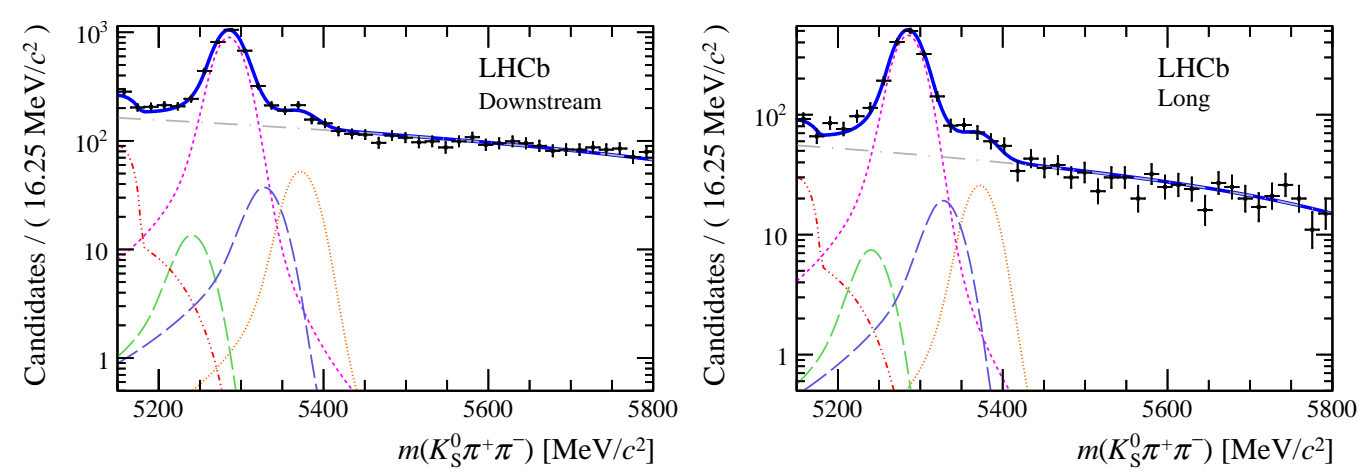

Figure 1: Invariant mass fit of decays reconstructed in the $K_{\mathrm{S}}^{0} \pi^{+} \pi^{-}$, for $K_{\mathrm{S}}^{0}$ mesons reconstructed from two downstream tracks (left), and two long tracks (right). The $B^{0} \rightarrow K_{\mathrm{S}}^{0} \pi^{+} \pi^{-}$signal component is indicated in magenta, the $B_{s}^{0} \rightarrow K_{\mathrm{S}}^{0} \pi^{+} \pi^{-}$signal component is indicated in orange, and all other colours indicate various sources of background.

$$
\begin{aligned}
\mathscr{B}\left(B_{s}^{0} \rightarrow \bar{K}^{0} K^{ \pm} \pi^{\mp}\right) & =(84.3 \pm 3.5 \pm 7.4 \pm 3.4) \times 10^{-6}, \\
\mathscr{B}\left(B_{s}^{0} \rightarrow K^{0} K^{+} K^{-}\right) & \in[0.4-2.5] \times 10^{-6} \text { at } 90 \% \text { C.L. },
\end{aligned}
$$

Here the first uncertainty is statistical, the second is systematic, and the last is due to the uncertainty on the $B^{0} \rightarrow K^{0} \pi^{+} \pi^{-}$branching fraction. The yield of the $B_{s}^{0} \rightarrow K_{\mathrm{S}}^{0} K^{+} K^{-}$decay is consistent with zero at the level of $2.5 \sigma$, where $90 \%$ confidence interval for the branching fraction of this mode is calculated using the Feldman-Cousins prescription. These results are in agreement with, and more precise than, the current world-average, and supersede previous measurements of these quantities made by $\mathrm{LHCb}$ with $1 \mathrm{fb}^{-1}$ of data.

\section{Observation of $C P$ violation in $\bar{B}^{0} \rightarrow K^{*}(892)^{-} \pi^{+}$decays}

A time-integrated amplitude analysis is performed on $\bar{B}^{0} \rightarrow K_{\mathrm{S}}^{0} \pi^{+} \pi^{-}$decays, using $3 \mathrm{fb}^{-1}$ of Run $1 \mathrm{LHCb}$ data [5], where the data sample, selection procedure, and efficiency calculation procedure is identical to that described above for the $B_{(s)}^{0} \rightarrow K_{\mathrm{S}}^{0} h^{+} h^{-}$decay branching fraction analysis. The total decay amplitude is written as the incoherent sum of the $B^{0}$ and $\bar{B}^{0}$ decay amplitudes, where each of these is described by the sum of identical resonant and non-resonant components, multiplied by coefficients that are free to vary between the $B^{0}$ and $\bar{B}^{0}$ amplitudes for contributions that describe or interfere strongly with (quasi-)flavour-specific processes, such as for $B^{0} \rightarrow K^{*}(892)^{-} \pi^{+}$decays.

The amplitude model is constructed by considering components included in previous analyses, and additional components are included based on several likelihood ratio and fit quality criteria. This contains $K *(892)^{-}, K_{2}^{*}(1430)^{-}, f_{0}(500), f_{0}(1500)$, and $\chi_{c 0}$ resonant components with relativistic Breit-Wigner lineshapes; $K^{*}(1680)^{-}$and $f_{0}(980)$ with Flatté lineshapes; a $\rho(770)$ component with a Gounaris-Sakurai lineshape; and a $(K \pi)_{0}^{-}$S-wave contribution using the EFKLLM model. The projections of this model, when fitted to the data, can be seen in Figure 2.

Quasi-two-body $C P$ asymmetries are calculated for the set of contributions where the coefficients of the $B^{0}$ and $\bar{B}^{0}$ model are free to vary, and are found to be

$$
\mathscr{A}_{\mathrm{CP}}\left(K^{*}(892)^{-} \pi^{+}\right)=-0.308 \pm 0.060 \pm 0.011 \pm 0.012,
$$



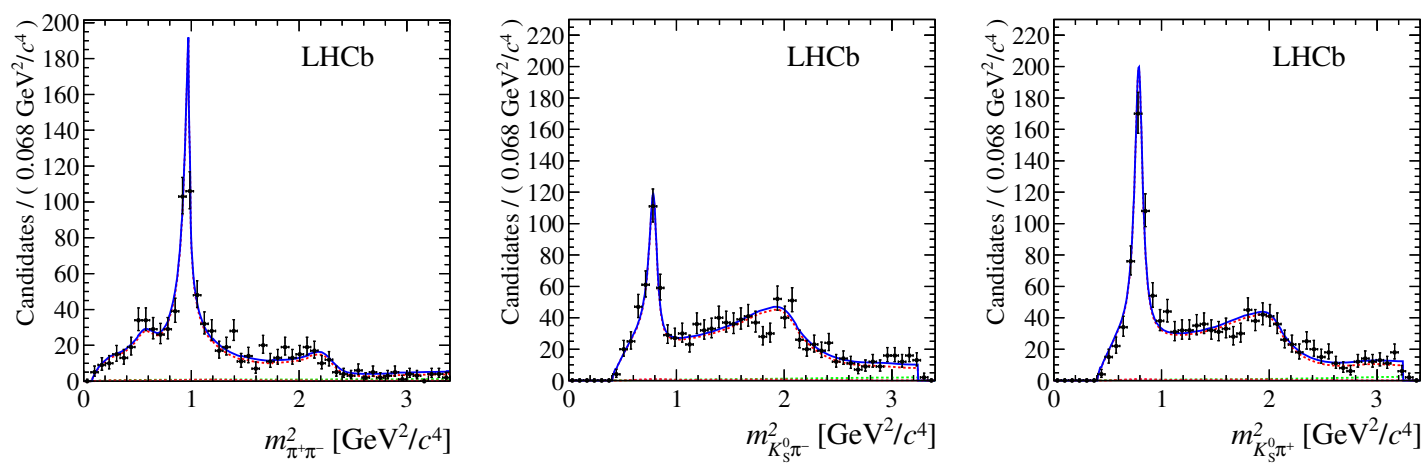

Figure 2: Projections of the fit model (blue) and data (points with error bars) on $m_{\pi^{+} \pi^{-}}$(left), $m_{K_{\mathrm{S}}^{0} \pi^{-}}$(centre) and $m_{K_{\mathrm{S}}^{0} \pi^{+}}$(right). The signal model is indicated in orange, the combinatorial background in green, and the cross-feed background in red.

$$
\begin{aligned}
\mathscr{A}_{\mathrm{CP}}\left((K \pi)_{0}^{-} \pi^{+}\right) & =-0.032 \pm 0.047 \pm 0.016 \pm 0.027, \\
\mathscr{A}_{\mathrm{CP}}\left(K_{2}^{*}(1430)^{-} \pi^{+}\right) & =-0.29 \pm 0.22 \pm 0.09 \pm 0.03, \\
\mathscr{A}_{\mathrm{CP}}\left(K^{*}(1680)^{-} \pi^{+}\right) & =-0.07 \pm 0.13 \pm 0.02 \pm 0.03, \\
\mathscr{A}_{\mathrm{CP}}\left(f_{0}(980) K_{\mathrm{S}}^{0}\right) & =0.28 \pm 0.27 \pm 0.05 \pm 0.14,
\end{aligned}
$$

where the first uncertainty is statistical, and the last two are systematic. In particular, $C P$ violation is observed for the first time in $\bar{B}^{0} \rightarrow K^{*}(892)^{-} \pi^{+}$decays, with a significance of approximately $6 \sigma$, and a value consistent with the world-average [4].

\section{Summary}

Measurements of the branching fractions of the $B_{(s)}^{0} \rightarrow K_{\mathrm{S}}^{0} h^{+} h^{-}$decays are presented, obtained relative to the $B^{0} \rightarrow K^{0} \pi^{+} \pi^{-}$decay branching fraction, and limits are set on contributions from the $B_{s}^{0} \rightarrow K^{0} K^{+} K^{-}$decay. Furthermore, results are presented of an amplitude analysis of $\bar{B}^{0} \rightarrow$ $K_{\mathrm{S}}^{0} \pi^{+} \pi^{-}$decays, where $C P$ violation in the $\bar{B}^{0} \rightarrow K^{*}(892)^{-} \pi^{+}$decay is observed for the first time with a significance of around $6 \sigma$.

\section{References}

[1] LHCb collaboration, A. A. Alves Jr. et al., The LHCb detector at the LHC, JINST 3 (2008) S08005

[2] LHCb collaboration, R. Aaij et al., Updated branching fraction measurements of $B_{(s)}^{0} \rightarrow K_{\mathrm{S}}^{0} h^{+} h^{\prime-}$, JHEP 11 (2017) 027, arXiv:1707.01665.

[3] M. Pivk and F. R. Le Diberder, sPlot: A statistical tool to unfold data distributions, Nucl. Instrum. Meth. A555 (2005) 356, arXiv: physics $/ 0402083$.

[4] Particle Data Group, M. Tanabashi et al., Review of particle physics, Phys. Rev. D98 (2018) 030001

[5] LHCb collaboration, R. Aaij et al., Amplitude analysis of the decay $\bar{B}^{0} \rightarrow K_{\mathrm{S}}^{0} \pi^{+} \pi^{-}$and first observation of the CP asymmetry in $\bar{B}^{0} \rightarrow K^{*}(892)^{-} \pi^{+}$, Phys. Rev. Lett. 120, 261801 (2018), arXiv: 1712.09320. 